BMJ Open

Respiratory

Research

\section{Randomised placebo-controlled cross- over study examining the role of anamorelin in mesothelioma (The ANTHEM study): rationale and protocol}

To cite: Hoon SN, Fyfe K, Peddle-McIntyre CJ, et al. Randomised placebo-controlled cross-over study examining the role of anamorelin in mesothelioma (The ANTHEM study): rationale and protocol. BMJ Open Resp Res 2020;7:e000551. doi:10.1136/ bmjresp-2019-000551

Received 19 December 2019 Revised 31 January 2020 Accepted 9 February 2020

\section{Check for updates}

\section{(c) Author(s) (or their} employer(s)) 2020. Re-use permitted under CC BY-NC. No commercial re-use. See rights and permissions. Published by BMJ.

For numbered affiliations see end of article.

Correspondence to Professor Fraser Brims; fraser.brims@curtin.edu.au

\section{ABSTRACT}

Introduction Cachexia is common in malignant mesothelioma (MM); half of patients have malnutrition and low skeletal muscle mass. Malnourished patients have worse quality of life (QoL). Weight loss is strongly associated with poor survival. Anamorelin is an oral ghrelin receptor agonist that improves appetite, body weight and QoL in advanced cancer. The aim of this study is to examine the efficacy of anamorelin in improving appendicular skeletal muscle mass (ASM) and patientreported outcomes in patients with MM with cachexia. Methods and analysis A single-centre, phase II, randomised, placebo-controlled cross-over pilot study with 28-day treatment periods and 3-day washout. Forty patients will be randomised. Primary outcome is change in ASM relative to height measured by dual energy X-ray absorptiometry at end of period 1. Secondary outcomes include cancer-specific and cachexia-related QoL, objective physical activity, dietary intake and adverse events. Eligible patients will have confirmed MM, Eastern Cooperative Oncology Group 0-2, expected survival $>3$ months and cachexia (defined as $>5 \%$ weight loss in 6 months or body mass index $<20 \mathrm{~kg} / \mathrm{m}^{2}$ with weight loss $>2 \%$ ).

Ethics and dissemination Ethical approval has been granted. Results will be reported in peer-reviewed publications.

Trial registration number Australian New Zealand Clinical Trials Registry (U1111-1240-6828).

\section{INTRODUCTION}

Malignant pleural mesothelioma (MPM) is a rare and universally fatal cancer developing from the mesothelial monolayer of the thoracic and abdominal cavities ${ }^{1}$ and has a median survival of 9-12 months. ${ }^{2}$ Treatment is palliative, with combination chemotherapy providing only a modest improvement in overall survival. ${ }^{3}$ Australia has one of the highest rates of mesothelioma per capita worldwide due to asbestos mining and use. In 2017, the age-standardised rate of mesothelioma in Australia was 2.7/100 000

\section{Key messages}

Our protocol details the design of a study to examine the efficacy of anamorelin in improving appendicular skeletal muscle mass and patient-reported outcomes in patients with malignant mesothelioma with cachexia.

- Anorexia is a major symptom in mesothelioma, and weight loss and cachexia are common and have prognostic importance, as such, management of anorexia is key in optimising both quality of life and survival.

- We believe our study is timely and relevant in a cohort of patients of which cachexia remains a prominent issue and that our protocol proposes the use of an agent that has been minimally used in the mesothelioma population.

population, an increase of $123 \%$ since 1982 , with 779 deaths nationally. ${ }^{4}$ Pleural mesothelioma accounts for $\sim 95 \%$ of all mesothelioma cases. $^{56}$

Weight loss, low muscle mass and malnutrition are common in patients with MPM and these are associated with poor quality of life (QoL), physical functioning and perhaps lower survival. A recent study examining patients with MPM found that high rates of malnutrition (38\%) and low skeletal muscle mass $(54 \%)$ were present shortly after diagnosis (median 2 months). ${ }^{7}$ Participants with malnutrition had lower QoL $(p<0.001)$, while participants with low skeletal muscle mass were more inactive compared with those with normal muscle mass $(p=0.001) .^{7}$ Additionally, participants with malnutrition and had significantly higher levels of the proinflammatory cytokine, interleukin (IL) 6, compared with well-nourished participants (median 11.0 (IQR $4.7-18.8$ ) $\mathrm{pg} / \mathrm{mL}$ versus median 
$2.6(2.0-7.9) \mathrm{pg} / \mathrm{mL} ; \mathrm{p}=0.002) .{ }^{7}$ These results suggest the presence of systemic inflammation in our malnourished participants with MPM and are indicative of cancer cachexia rather than simple malnutrition.

Numerous studies have shown that weight loss may be directly related to worse survival in MPM. ${ }^{2}{ }^{8-10}$ A postmortem study of 318 patients with MPM found that body mass index (BMI) was significantly lower where there was no identifiable anatomic cause of death. ${ }^{8}$ The mean BMI of this cohort was 19.98 (SD 4.9) and BMI was significantly lower in patients with no identified anatomic cause of death, compared with those where causes were apparent (18.8 (SD 4.3) vs 21.0 (4.7); $\mathrm{p}=0.034){ }^{8}$ A recent prognostic model for MPM was consistent with this notion, demonstrating that the presence of weight loss at the time of diagnosis of MPM has the strongest association with survival. ${ }^{2}$ Additionally, post-hoc analyses of the Regular Early Specialist Palliative Care Mesothelioma (RESPECT-Meso) study also demonstrated that loss of appetite was associated with worse survival (HR 2.3 (95\% CI 1.2 to $4.4, \mathrm{p}=0.01)) .{ }^{9}{ }^{10}$ Overall, these findings suggest any interventions that can potentially reduce weight loss or improve appetite associated with advanced cancer may positively impact on QoL, physical functioning and, perhaps, survival.

Current treatment options for cancer cachexia are very limited. Oral corticosteroids and progestins can improve appetite and total body weight (TBW) but have no beneficial effect on skeletal muscle mass. ${ }^{11}$ These drugs pose a significant risk of side effects that can impact multiple body symptoms. ${ }^{12}$ Ghrelin receptor agonists have been developed as a treatment for cancer cachexia. Ghrelin is predominantly produced in the stomach and rapidly stimulates appetite leading to increased food intake. Ghrelin also triggers other physiological changes such as stimulation of gastric emptying and increased skeletal muscle, probably from inducing growth hormone secretion and insulin-like growth factor (IGF)-1 release. ${ }^{13}$

Anamorelin is a ghrelin receptor agonist and in advanced cancers it improves TBW, lean body mass (LBM), appetite and QoL. ${ }^{14-17}$ A recent systematic review of the effects of anamorelin identified 6 randomised controlled trials of 1641 participants. ${ }^{18}$ These comprised two phase III trials in in non-small cell lung cancer (NSCLC) and four phase II studies with mixed cancer cohorts. In these studies, there is a consistent treatment effect favouring anamorelin, with statistically significant improvements in TBW, LBM and QoL. ${ }^{18}$ Since the systematic review, there has been a further phase III study trialling anamorelin in NSCLC and a phase II study in mixed gastrointestinal cancers (mainly colorectal). ${ }^{19} 20$ The phase III study in NSCLC demonstrated a mean increase in LBM by $1.38 \mathrm{~kg} \pm 0.18(\mathrm{p}<0.001)$ with associated increases in body weight $(1.06 \mathrm{~kg} \pm 0.2, \mathrm{p}<0.0001)$ and appetite $(\mathrm{p}=0.0005)$, compared with placebo over a 12-week period. ${ }^{19}$ The participants with gastrointestinal cancer in the phase II study demonstrated a similar increase in LBM of $1.89 \mathrm{~kg}$ \pm 0.36 , and increase in appetite. ${ }^{20}$
The majority of these studies administered anamorelin to participants over a 12-week period. In responders, a pattern of rapid improvement in appetite and weight occurs within the first 3 weeks which progresses to sustained benefit for the duration of the studies. 15171920 Improvement in cachexia-related biomarkers collected throughout these studies followed a similar pattern, with biomarkers such as IGF-1, IGF3, IGF-binding protein 3 , prealbumin and transthyretin increasing to a peak at week 3 or 4 , then plateauing or decreasing after this point. ${ }^{15171920}$

Despite the clear promise as a palliative therapeutic agent in both improvement in weight and QoL, anamorelin has not had regulatory approval, in part, because coprimary outcome measures were not significantly improved in the two large, phase III anamorelin in lung cancer cachexia (ROMANA) studies. ${ }^{17}$ The Federal Drugs Administration requirement for inclusion of hand grip strength as a coprimary outcome measure. These studies, with 979 participants, demonstrated a clear statistically significant benefit in LBM favouring anamorelin as well as a consistent improvement in appetite and QoL but failed the coprimary endpoint on hand grip strength. ${ }^{17}$ In our studies, we have found that hand grip strength is not sensitive to change in our MPM population in both observational and interventional cohorts. ${ }^{21}$

Anamorelin is well tolerated with no dose-limiting toxicities identified to date; the frequency of grade 3 and 4 toxicities is the same as for patients receiving placebo. ${ }^{18}$ Hyperglycaemia is the most common highgrade toxicity but occurs in $<1 \%$ of participants. ${ }^{17}$ Mild nausea and abnormal liver function tests are the other most frequently reported adverse events (AE) in up to 1:10 patients. There have been concerns in early phase clinical studies about cardiovascular toxicity, but this was not borne out in later phase studies, with the rates of cardiovascular events being similar between anamorelin and placebo. ${ }^{17}$ Two dose levels of anamorelin have been examined (50 mg and $100 \mathrm{mg}$ daily), with the $100 \mathrm{mg}$ dose considered optimal to improve skeletal muscle mass without any increase in $\mathrm{AE}$.

Anamorelin has yet to be examined in patients with MPM. The aim of this randomised controlled trial is to assess if anamorelin improves muscle mass and QoL in patients with MPM as compared with placebo.

\section{METHODS AND ANALYSIS \\ Primary aim}

The primary aim of this trial is to examine the efficacy of anamorelin in improving appendicular skeletal muscle mass (ASM) relative to height in cachectic patients with MPM. This will be measured at 28 days by dual-energy X-ray absorptiometry (DXA; Hologic Discovery A, Hologic, Marlborough, Massachusetts, USA) scan. ASM has been chosen as a measure of skeletal muscle mass, as it represents approximately $75 \%$ of total skeletal muscle 
and has shown to correlate well with nutrition and physical status. ${ }^{722}$

\section{Secondary aims}

The secondary aims include assessment of ASM at 60 days and overall changes in weight, objective physical activity, physical functioning and dietary intake. Each of these measurements will be taken at baseline, 28 days and 60 days. Cancer-specific and cachexia-related QoL will be measured using the Functional Assessment Cancer Therapy-Lung (FACT-L) ${ }^{23}$ and Anorexia Cachexia Scale (ACS) instruments at the same time points. ${ }^{24}$

Objective activity behaviours will be assessed at each time point Actigraph GT3X+accelerometer (Actigraph, Pensacola, Florida, USA). Participants will be instructed to wear the accelerometer on their hip continuously (24hours/day) for 3 days. ${ }^{7}$ Dietary intake will be measure by a 24 hours recall completed in an interview with a dietitian. Physical function will be objectively assessed using three different measures: repeated chair rise, gait speed and hand-grip strength. These physical functioning assessments are in accordance with current consensus documents regarding sarcopenia. ${ }^{25}$ Hand grip strength has been included to add applicability to other similar studies in malnutrition and sarcopenia, as well as to directly compare to previous studies with anamorelin. ${ }^{172627}$

Survival will be measured from the date of randomisation to death from any cause, or censure at end of study (12 weeks after last participant randomisation). Adverse effects from anamorelin will be actively monitored throughout the course of the study at the faceto-face participant visits and during planned telephone interactions. Patient preference for the first or second study period will also be assessed by a study-specific questionnaire.

\section{Exploratory aims}

Correlative biomarkers of anorexia, cachexia and nutrition will be examined to assess if there is a change in response to anamorelin. These include inflammationassociated factors in the serum: interferon-gamma, IL-1 alpha, IL1 beta, IL-10, IL-13, IL-4, IL-6, IL-8, monocyte chemoattractant protein-1 and tumour necrosis factor alpha. Serum albumin, plasma glycerol, serum leptin, ghrelin, transthyretin and serum adiponectin will also be measured. The choice of correlative biomarkers is based on previous studies of cachexia in mesothelioma. ${ }^{728}$

\section{Box 1 Inclusion and exclusion criteria}

\section{Inclusion criteria}

- Adults (18 years and above)

- Histological or cytological confirmation of malignant pleural mesothelioma

Cachexia (defined as $>5 \%$ weight loss in 6 months or body mass index $<20 \mathrm{~kg} / \mathrm{m}^{2}$ with weight loss $>2 \%$ );

Life expectancy $>3$ months at randomisation,

Eastern Cooperative Oncology Group performance status 0-2,

- At least 3 weeks since last received systemic anticancer therapy

\section{Exclusion criteria}

$\checkmark$ Peritoneal disease

Other significant comorbidity or organ dysfunction which may affect outcome measures or safety,

- Impaired cardiac function or clinically significant cardiac diseases, including any of the following:

- History or presence of ventricular tachyarrhythmia

- Presence of unstable atrial fibrillation (ventricular response $>120$ beats/min at rest); patients with stable atrial fibrillation are eligible, provided they do not meet any of the other cardiac exclusion criteria

- Corrected QT interval (QTCF) $>480$ ms on baseline ECG.

- Patients with abnormal liver function tests, defined as any of the following:

- Aspartate aminotransferase (AST) or alanine aminotransferase (ALT)>3 times the upper limit of normal (ULN), or AST or ALT>5 times ULN for patients with liver metastases

- Total bilirubin $>1.5 \times$ ULN, except for patients with Gilbert's syndrome who are excluded if total bilirubin $>3.0 \times$ ULN or direct bilirubin $>1.5 \times$ ULN

- Participation in another study (drug or non-drug study) that may affect outcome measures

$\checkmark$ Concurrent chemotherapy or radiotherapy (immunotherapy is accepted), including planned chemotherapy or radiotherapy commencing during the study period

- Concurrent use of appetite stimulants including oral corticosteroids (other than for adrenal replacement), mirtazapine (other than for major depressive disorder), or progesterones

- Patients who are receiving treatment with medications, that cannot be discontinued prior to study entry and that are considered to be any of the following:

- known risk for QT prolongation

- known to be strong inducers or inhibitors of CYP3A4/5

Uncontrolled diabetes mellitus (defined as random blood glucose $>11.1 \mathrm{mmol} / \mathrm{L}$ and glycated haemoglobin $>7.0 \%$ )

- Significant active gastrointestinal disease that might impair absorption of study treatment

Inability to readily swallow oral tablets or intractable or frequent vomiting 


\section{Study design}

This is a single-centre, randomised double-blind phase II placebo-controlled modified cross-over study. Each phase comprises 28-day treatment separated by a 3-day washout. A $2 \times 28$ day period design has been selected as it is sufficient to demonstrate benefit but patient deterioration due to cancer progression should be modest over that period. The cross-over design has been selected as every participant will receive the intervention; this is not only very attractive for potential participants and their families (as per community feedback), but there will be reduced, between-patient variability, allowing greater precision in estimating the treatment effect. The 3-day washout period comprises at least 10 half-lives of the study drug, reducing the possibility of residual drug-effect in period 2. The potential bias of a carry-over effect on ASM from periods 1 to 2 has been considered; as such, the primary outcome will compare ASM between the groups at end of period 1 .

Given the expected dropout and the relatively small sample size, this study will adopt an adaptive-recruitment design that will allow further recruitment of additional participants in the event that withdrawal occurs in the period after randomisation and before baseline assessment, or a participant begins period 1 but withdraws before day 14 .

\section{Recruitment and informed consent}

Potential participants will be identified through outpatient clinics based at Sir Charles Gairdner Hospital, Perth, Western Australia. Community-based palliative care physicians will also be encouraged to refer potential participants. Suitable patients will be provided with a verbal and written explanation of the study in the form of an information sheet, and informed consent will be obtained by a member of the research team in accordance with good clinical practice (GCP). The inclusion and exclusion criteria are listed in box 1 .

\section{Randomisation and blinding}

Participants will be minimised with a random element with a 1:1 ratio between the two groups using an online computer-generated randomisation sequence through the REDCap (REDCap V.9.3.0-2019 Vanderbilt University) program which will be managed by Curtin Clinical Trials and Data Management Centre (CCTDMC) in Bentley, Australia. Minimisation will be according to: Eastern Cooperative Oncology Group performance status (0-1 or 2), and histological subtype (epithelioid vs nonepithelioid (biphasic, sarcomatoid, not defined)). This is a double-blind study and assessors of outcomes will also be blinded; however, there will be no independent review of assessments.

\section{Study intervention and control arm}

Patients will be randomised to receive either anamorelin $100 \mathrm{mg}$ oral tablet once daily in the first period or placebo. As per the cross-over design, those randomised to anamorelin in the first period will receive placebo in the second period, and vice versa. Participants will be instructed to take the study drug (either anamorelin or placebo) on an empty stomach, at approximately the same time each day throughout the study. Both anamorelin and the placebo will be packaged and labelled identically in accordance with GCP. All participants will receive all other appropriate standard care management as required.

\section{Study visit schedule}

The participant visit schedule consists of face-to-face and telephone interactions as detailed in table 1. After consent, the following information will be collected from participants at a first face-to-face visit; demographics

\begin{tabular}{|c|c|c|c|c|c|c|c|c|c|c|c|}
\hline \multirow[b]{2}{*}{ Assessment } & \multirow{2}{*}{$\begin{array}{l}\text { Baseline } \\
\text { Do }\end{array}$} & \multicolumn{4}{|l|}{ Period 1} & \multirow{2}{*}{$\begin{array}{l}\text { Washout/ } \\
\text { second } \\
\text { visit }\end{array}$} & \multicolumn{4}{|l|}{ Period 2} & \multirow{2}{*}{$\begin{array}{l}\begin{array}{l}\text { Final } \\
\text { visit }\end{array} \\
\text { D61 } \\
( \pm 3) \\
\end{array}$} \\
\hline & & D1 & $\begin{array}{l}\text { D14 } \\
( \pm 1)\end{array}$ & D26 & D28 & & D32 ( \pm 3$)$ & $\begin{array}{l}\text { D46 } \\
( \pm 1)\end{array}$ & D58 & D60 & \\
\hline $\begin{array}{l}\text { Blood collection } \\
\text { (biomarker analysis, } \\
\text { safety blood tests) }\end{array}$ & $x$ & $\begin{array}{l}\text { First dose of } \\
\text { study drug/ } \\
\text { placebo for }\end{array}$ & $x$ & & $\begin{array}{l}\text { Final dose of } \\
\text { study drug/ } \\
\text { placebo for }\end{array}$ & $x$ & $\begin{array}{l}\text { First dose of } \\
\text { study drug/ } \\
\text { placebo for }\end{array}$ & $x$ & & $\begin{array}{l}\text { Final dose of } \\
\text { study drug/ } \\
\text { placebo for }\end{array}$ & $x$ \\
\hline Weight & $x$ & & & & & $x$ & & & & & $x$ \\
\hline ECG & $x$ & & & & & $x$ & & & & & $x$ \\
\hline QoL: FACT-L and ACS & $x$ & & & & & $x$ & & & & & $x$ \\
\hline Accelerometer & $X^{*}$ & & & $\mathrm{X}^{*}$ & & & & & $\mathrm{X}^{*}$ & & \\
\hline 24 hours dietary recall & $x$ & & & & & $x$ & & & & & $x$ \\
\hline
\end{tabular}

*Wear accelerometer.

ACS, Anorexia Cachexia Scale; DXA, dual X-ray absorptiometry; FACT-L, Functional Assessment of Cancer Therapy-Lung; QoL, quality of life. 
including treatment history, medications and comorbidities, histological subtype of mesothelioma; QoL questionnaires (FACT-L, ACS); blood tests (haematological, renal, hepatic function, blood glucose (blood sugar level (BSL)), ECG; objective physical functioning (repeated chair rise, gait speed and hand-grip strength); exploratory biomarkers; height; weight; vital signs; physical examination and a DXA scan. Each participant will also be given an accelerometer to wear for 3 days. They will also be contacted by a dietician to complete a 24 hours food recall. The participant will be instructed to commence taking the study drug (anamorelin or placebo) on the day after this visit.

At day 14 and day 46, participants from both groups will be contacted by telephone to ensure that they have performed a safety blood test to assess hepatic function and BSL and check on AEs or non-compliance. They will receive an accelerometer in the mail and be instructed to wear this starting 3 days prior to the next scheduled visit.

Participants will complete 28 days of treatment followed by a 3-day washout. At the end of both periods, participants will return for a face-to-face visit with further assessments including but not limited to: AEs or non-compliance; QoL questionnaires; safety blood tests; ECG; objective physical activity tests; exploratory biomarkers; weight and DXA scan. They will be contacted again by a dietician to complete a 24 hours food recall. At the end of the first period, participants will be instructed to commence the second period of study drug, the day after the face-to-face assessment. After completion of the second period and face to face visit, there will be no further follow-up.

\section{Safety monitoring and reporting}

There will be close monitoring for any potential $\mathrm{AE}$ or serious adverse events (SAE) from anamorelin. Participants may be withdrawn from the trial if they develop an $\mathrm{AE}$ of grade 3 or above or SAE related to study drug, with mandatory reporting to the therapeutic drugs administration as required. For this small study, the Data Safety Monitoring Board and Trial Steering Committee will be combined.

\section{Sample size}

Based on previous research, an improvement in ASM of $0.2 \mathrm{~kg} / \mathrm{m}^{2}$ is considered significant for patients with thoracic cancer taking anamorelin. ${ }^{17}$ Pilot data demonstrate that up to $5 \%$ of the control group may have a $0.2 \mathrm{~kg} / \mathrm{m}^{2}$ increase in ASM adjusted for height over an 8 -week period. With $50 \%$ of the participants in the intervention group expected to have a $0.2 \mathrm{~kg} / \mathrm{m}^{2}$ increase in ASM adjusted for height, 15 participants per group will be required to detect the difference with $80 \%$ power at $5 \%$ significance level. ${ }^{17}$ To allow for a $25 \%$ dropout rate, ${ }^{18}$ a total sample size of 40 participants is required.

\section{Statistics}

All study data will be managed by CCTDMC using a bespoke database created using REDCap. Confidentiality of participant data will be assured according to GCP. All participants will be included for an intention-to-treat analysis. Sensitivity analyses will be preplanned to include only those participants who continued study treatment for greater than 14 days. All participants who take one dose or more of study drug will be included in analyses of toxicity.

\section{Clinical outcomes analysis}

Differences between ASM (and other secondary endpoints) at baseline and across the follow-up time points will be assessed using general linear mixed effects model. The model will allow comparison within participants over time and between the groups, while allowing a time by group interaction (ie, 'period' and 'intervention'). Analysis for the presence of a period effect and/or carry-over effect will be performed with a null hypothesis of no difference between periods 1 and 2 .

As the intervention may influence the presence (or absence) of missing data, we will not assume that data are missing at random, instead, the joint modelling approach detailed above will be used to assess the robustness of any assumptions with missing data. Survival curves of the two groups will be estimated using the Kaplan-Meier method and compared statistically using the log rank test. Covariates for the mixed effects submodel will include baseline measurements of the outcome, treatment group, measurements at each time point and a treatment groupmeasurement time point interaction. Sensitivity analyses will include physical activity and dietary intake data. Correlation between biomarkers of cachexia and major nutrients will be assessed using linear regression while adjusting for total energy intake.

\section{Patient and public involvement}

Our consumer reference group has been involved in the development of this protocol, review of patient information consent form and the recruitment materials.

\section{Timelines and trial status}

This study plans to open to recruitment in early 2020 and is expected to recruit for 12-18 months.

\section{ETHICS AND DISSEIMINATION}

A favourable ethical approval for this study has been granted by Sir Charles Gairdner Osborne Park Health Group Human Research Ethics Committee (Ref\# 3154) and has been registered on Australian New Zealand Clinical trials registry (U1111-1240-6828).

The study results will be disseminated via clinical and scientific conferences and peer-reviewed publications in scientific journals. A lay summary will be generated for patients and consumers. 


\section{CONCLUSION}

Anorexia is a major symptom in MPM, and weight loss and cachexia are common and have prognostic importance. People with cachexia are less likely to complete active therapy and more likely to experience toxicities. Better management of anorexia may improve QoL and ASM. In the design of the ANTHEM study, we have attempted to allow as pragmatic an approach as possible, to ensure as wide as possible external applicability of the results, within the confines of ensuring internal validity and appropriate scientific rigour. Larger clinical trials will be needed to test the effects of anamorelin on survival or treatment toxicities and adherence.

\section{Author affiliations}

${ }^{1}$ Respiratory Medicine, Sir Charles Gairdner Hospital, Nedlands, Western Australia, Australia

${ }^{2}$ Curtin Medical School, Curtin University, Perth, Western Australia, Australia ${ }^{3}$ Institute of Respiratory Health, Nedlands, Western Australia, Australia ${ }^{4}$ Exercise Medicine Research Institute, Edith Cowan University—Joondalup Campus, Joondalup, Western Australia, Australia

${ }^{5}$ Medical Oncology, Sir Charles Gairdner Hospital, Nedlands, Western Australia, Australia

${ }^{6}$ Palliative Care, Sir Charles Gairdner Hospital, Nedlands, Western Australia, Australia

${ }^{7}$ School of Medicine and Pharmacology, University of Western Australia, Perth, Western Australia, Australia

${ }^{8}$ School of Public Health, Curtin University, Perth, Western Australia, Australia

Contributors SNH, KF and AN: study design, protocol development. SB and FH: study design. EJ: study design, protocol development, dietary interviews. HJC: statistical expertise. JC: protocol development, biomarker analysis. CJP-M: study design, protocol development, exercise physiology. FB: study lead, study design, protocol development.

Funding This project is supported by a competitive grant from the Cancer Council Western Australia (Ref RES-HEA-CMS-KC-59836-1) and the Western Australian Cancer and Palliative Care Network. The sponsor for this trial is the Institute of Respiratory Health in collaboration with SCGH.

Competing interests None declared.

Patient and public involvement Patients and/or the public were involved in the design, or conduct, or reporting, or dissemination plans of this research. Refer to the Methods section for further details.

Patient consent for publication Not required.

Provenance and peer review Not commissioned; externally peer reviewed.

Open access This is an open access article distributed in accordance with the Creative Commons Attribution Non Commercial (CC BY-NC 4.0) license, which permits others to distribute, remix, adapt, build upon this work non-commercially, and license their derivative works on different terms, provided the original work is properly cited, appropriate credit is given, any changes made indicated, and the use is non-commercial. See: http://creativecommons.org/licenses/by-nc/4.0/.

\section{REFERENCES}

1 Robinson BWS, Lake RA. Advances in malignant mesothelioma. $N$ Engl J Med 2005;353:1591-603.

2 Brims FJH, Meniawy TM, Duffus I, et al. A novel clinical prediction model for prognosis in malignant pleural mesothelioma using decision tree analysis. J Thorac Oncol 2016;11:573-82.

3 Vogelzang NJ, Rusthoven JJ, Symanowski J, et al. Phase III study of pemetrexed in combination with cisplatin versus cisplatin alone in patients with malignant pleural mesothelioma. $J$ Clin Oncol 2003;21:2636-44.

4 AlHW. Cancer in Australia 2017. Canberra: Australian Institute of Health and Welfare, 2017.

5 Robinson BWS, Musk AW, Lake RA. Malignant mesothelioma. Lancet 2005;366:397-408.
6 Muruganandan S, Alfonso H, Franklin P, et al. Comparison of outcomes following a cytological or histological diagnosis of malignant mesothelioma. Br J Cancer 2017:116:703-8.

7 Jeffery E, Lee YCG, Newton RU, et al. Body composition and nutritional status in malignant pleural mesothelioma: implications for activity levels and quality of life. Eur J Clin Nutr 2019;73:1412-21.

8 Finn RS, Brims FJH, Gandhi A, et al. Postmortem findings of malignant pleural mesothelioma: a two-center study of 318 patients. Chest 2012:142:1267-73.

9 Brims F, Gunatilake S, Lawrie I, et al. Early specialist palliative care on quality of life for malignant pleural mesothelioma: a randomised controlled trial. Thorax 2019;74:354-61.

10 Brims F, Lawire I, Qi C, et al. The Relationship of Quality of Life, Physical Symptoms and Survival in Mesothelioma - Exploratory Analyses from the Respect-Meso Study. Am J Respir Crit Care Med 2019:A1247-A47.

11 Aoyagi T, Terracina KP, Raza A, et al. Cancer cachexia, mechanism and treatment. World J Gastrointest Oncol 2015;7:17-29.

12 Stanbury RM, Graham EM. Systemic corticosteroid therapy--side effects and their management. Br J Ophthalmol 1998;82:704-8.

13 Zhang H, Garcia JM. Anamorelin hydrochloride for the treatment of cancer-anorexia-cachexia in NSCLC. Expert Opin Pharmacother 2015;16:1245-53.

14 Garcia JM, Friend J, Allen S. Therapeutic potential of anamorelin, a novel, oral ghrelin mimetic, in patients with cancer-related cachexia: a multicenter, randomized, double-blind, crossover, pilot study. Support Care Cancer 2013;21:129-37.

15 Garcia JM, Boccia RV, Graham CD, et al. Anamorelin for patients with cancer cachexia: an integrated analysis of two phase 2, randomised, placebo-controlled, double-blind trials. Lancet Oncol 2015;16:108-16.

16 Takayama K, Katakami N, Yokoyama T, et al. Anamorelin (ONO7643) in Japanese patients with non-small cell lung cancer and cachexia: results of a randomized phase 2 trial. Support Care Cancer 2016;24:3495-505.

17 Temel JS, Abernethy AP, Currow DC, et al. Anamorelin in patients with non-small-cell lung cancer and cachexia (ROMANA 1 and ROMANA 2): results from two randomised, double-blind, phase 3 trials. Lancet Oncol 2016;17:519-31.

18 Nishie K, Yamamoto S, Nagata C, et al. Anamorelin for advanced non-small-cell lung cancer with cachexia: systematic review and meta-analysis. Lung Cancer 2017;112:25-34.

19 Katakami N, Uchino J, Yokoyama T, et al. Anamorelin (ONO-7643) for the treatment of patients with non-small cell lung cancer and cachexia: results from a randomized, double-blind, placebocontrolled, multicenter study of Japanese patients (ONO-7643-04). Cancer 2018:124:606-16.

20 Hamauchi S, Furuse J, Takano T, et al. A multicenter, openlabel, single-arm study of anamorelin (ONO-7643) in advanced gastrointestinal cancer patients with cancer cachexia. Cancer 2019;125:4294-302.

21 McIntyre C. Exercise in Mesothelioma - as part of " Exercise Training To Improve Care for Patients with Malignant Pleural Disease" Research Grant:2018-20.

22 Rathnayake N, Alwis G, Lenora J, et al. Concordance between appendicular skeletal muscle mass measured with DXA and estimated with mathematical models in middle-aged women. $J$ Physiol Anthropol 2018;37:19.

23 Cella DF, Bonomi AE, Lloyd SR, et al. Reliability and validity of the functional assessment of cancer Therapy-Lung (FACT-L) quality of life instrument. Lung Cancer 1995;12:199-220.

24 LeBlanc TW, Samsa GP, Wolf SP, et al. Validation and real-world assessment of the functional assessment of anorexia-cachexia therapy (FAACT) scale in patients with advanced non-small cell lung cancer and the cancer anorexia-cachexia syndrome (CACS). Support Care Cancer 2015;23:2341-7.

25 Cruz-Jentoft AJ, Bahat G, Bauer J, et al. Sarcopenia: revised European consensus on definition and diagnosis. Age Ageing 2019;48:16-31.

26 Kilgour RD, Vigano A, Trutschnigg B, et al. Handgrip strength predicts survival and is associated with markers of clinical and functional outcomes in advanced cancer patients. Support Care Cancer 2013;21:3261-70.

27 Prado CMM, Lieffers JR, Bowthorpe L, et al. Sarcopenia and physical function in overweight patients with advanced cancer. Can $J$ Diet Pract Res 2013;74:69-74.

28 Nowak AK, Stockler MR, Byrne MJ. Assessing quality of life during chemotherapy for pleural mesothelioma: feasibility, validity, and results of using the European organization for research and treatment of cancer core quality of life questionnaire and lung cancer module. J Clin Oncol 2004;22:3172-80. 